THEORIA ET HISTORIA SCIENTIARUM, VOL. VIII, $\mathrm{N}^{\circ} 1$

Ed. Nicolaus Copernicus University 2008

Daniel Rothbart

\title{
Scientific Prototypes as Analogies of Nature
}

Thomas Kuhn inaugurated a resurgence of interest in the evolution of scientific ideas (1962; 1970; 1977). Just below the surface of his conception of paradigm- driven science is a provocative but undeveloped understanding of scientific analogies. The commitment to a new paradigm is often inspired, and defined, by the creative insights from analogical association of ideas. During an era of extraordinary science, to use Kuhn's notion, intractable obstacles to progress can be overcome by analogical comparisons between distinct domains of inquiry, providing fertile ground for potentially profound scientific discoveries. But for many critics, scientific analogies are dismissed as mere heuristic fictions, designed to facilitate instruction for students and not to promote discovery by scientists (Bunge 1973). Yet, the historical treasures originating from analogy cannot be denied, and suggest a central role for analogy in the development of science. To mention a familiar example, contemporary biochemists have discovered how the bases of DNA molecules carry genetic information, and in so doing, explain biological phenomena in terms of information processes. Such analogies are now indispensable to genetics.

In this paper I explore how certain Kuhnian themes concerning the evolution of science are given a more nuanced reading through a study of scientific analogy. I argue that many scientific discoveries are inspired by analogical models (Section 1), that such models often function as prototypes (not paradigms) for developing a particular target system (Section 2), and that prototype modeling is particularly valuable for designing modern scientific instruments (Section 3). From this discussion of scientific analogy, we discover that the endorsement of prototype models often precipitates severe strains in the literal vocabulary at a particular 
time, necessitating metaphoric language (Section 4). Scientific analogy must be taken seriously for a philosophical understanding of the conceptual process for generating scientific ideas. The reward of such an understanding is a deeper conception of scientific progress than one finds in Kuhn's work and in much of the contemporary literature.

\section{What is an Analogical Model?}

What kinds of things do scientists examine? According to empiricist-oriented philosophers of science, discrete events, occurring in an unordered swirl of action and interaction, comprise the primary subject matter of science. On this view phenomena are understood atomistically, because the identity of one phenomenon can be achieved in isolation from that of another. Once scientists describe phenomena in observation reports, a lawlike regularity can be inferred using the principles of inductive logic.

Since the 1960's, critics of empiricism have challenged the possibility of theoryneutral evidence in science by arguing that descriptions of phenomena of nature are always "contaminated" with theoretical judgments. But in my opinion this criticism misses a more serious deficiency in empiricist epistemologies, one which centers on the kinds of "things" that comprise the subject matter of science. The belief that scientists examine a succession of singular events (facts) is one of the great myths of empiricistoriented epistemology (Harre 1970, pp. 6-7). The primary domain of inquiry is not an unordered sequences of (factual) events, but are structures of phenomena. Scientific knowledge is indirectly about the natural world of mountains, electrons, and diseases, but directly about structures underlying such entities. The episodes of water freezing, boiling and absorbing presuppose chemical structures, understood through the configuration of atoms subject to electrostatic forces.

One medium for conveying such knowledge is the theoretical model. Common to every scientific discipline, a theoretical model is a system of concepts which are intended to replicate abstract properties of known phenomena. The successful model is not true of the environment since no direct correspondence between statement and world is intended. The categories of truth and falsity are irrelevant. No perfect duplication of the environment is possible, because the model at best simulates real-world structures. Simplification, approximation and abstraction of the environment are inescapable features of modeling. For example the 1953 Watson-Crick theoretical model of DNA replicates the double helica structure, not as a summary of isolated "facts" but as an abstract simulation of biochemical structures.

A theoretical model should not be confused with a scale model of a physical system. A scale model provides a physical substitute for the terrain, whereas 
a theoretical model constitutes a conceptual representation. The famous ball-and- wire scale model which Watson and Crick constructed in the Cavendish Laboratory provides a physical analogue to their theoretical insights (theoretical model).

Also, a theoretical model is not a formal model. A formal model is any set of entities that satisfies the axioms of a formal system and their deductive consequences. But in most cases the variables and relations of a theoretical model are not reducible to the formalism of mathematics and logic. Information about the molecular composition of the Watson-Crick model of DNA is not exhausted by any particular formalism. For example, each chain in the double helix consists of a phosphate di-ester group, joining beta-D-deoxyribofuranose residues with 3'5' linkages.

The centrality of analogical modeling to science was advanced in the 1920 s by the English physicist Norman Campbell, who established a considerable following among British philosophers. According to Campbell, models comprise a theory's content, so that, a belief in a scientific theory requires commitment to its models. To comprehend the kinetic model of gas, for example, we visualize the behavior of molecules through conceptual simulations. Gas behaves as if it is composed of point-particles randomly moving in a vessel. Later generations of philosophers developed these themes in more detail (Black 1962, p. 239; Harre 1970, Chapter 2; Hesse 1966; E. McMullin 1967; M. Spector 1965, pp. 135ff; J. Swanson 1966, p. 306).

In her influential work, Mary Hesse argues that analogical modeling provides scientists with the conceptual apparatus for expanding theories to new frontiers. Through modeling, one can identify the known similarities (positive analogies) and known differences (negative analogies) between target system and donor (analogical) system. New avenues of research are found in the neutral analogies, which are unknown relations between target and donor systems. Such relations display an "open texture", inviting scientists to examine possible, but yet unexplored, properties of the target system. One strategy to remedy deficiencies in our understanding of some phenomena is to highlight analogical associations between donor and target models. A donor model functions as an idealized standard for transforming a target model, as if the donor is a conceptual filter through which the incomplete target model passes. By injecting components from a donor model into an inadequate target, scientists may provide a more robust understanding of a problematic system.

Is it necessary, or even desirable, for analogical models to be visualizable? The question has provoked considerable controversy throughout the history of science, as illustrated by the debate between Heinrich Hertz and Ludwig Boltzmann concerning Maxwell's equations. According to Hertz, logical/ mathematical structures provide the primary content of scientific knowledge. Maxwell's theory is Maxwell's system of equations. Hertz argued that physical 
reality is structured logically by an internal grammar that is portrayed mathematically (Wilson 1989). Boltzmann objected that the mathematical models advocated by Hertz are almost unimaginably complicated. Hertz gave us no indication of how electromagnetic processes should be conceived. For Boltzmann, Maxwell's theory is not identical to his equations, but is conveyed by visualizable models, prompting internal mental pictures, or thought-pictures which presumably represent phenomena (Nyhof 1988). But in my opinion a theoretical model does not always provoke thought-pictures. As a conceptual replication of phenomena, a theoretical model need not be visualizable, as evident in the models of quantum mechanics.

The impact of analogies in the advancement of science has been well documented. Analogical modeling inspired development of Newtonian mechanics (J. McGuire 1970; J. North 1980), Maxwell's electromagnetism (R. Kargon 1969; M. Hesse 1974), and subatomic physics (Miller 1986). Episodes of analogical modeling in biology are well known (Bonner 1953; G. Canguilhem 1963; T. Hall 1968). Discoveries in structural chemistry can be understood through analogy (E. Farber 1950). In some episodes of scientific development, analogical models are used to generate entirely new disciplines. The nineteenth century unification of biology and chemistry was advanced by the exploration of analogical associations (J. Brooke 1971, 1973, 1980, 1987).

Analogical modeling is particularly important for developing causal explanations. To explain why certain events occurred, scientists often show that a causal mechanism of some physical or chemistry system was activated in ways that produced the events. Phenomena "come into being", as it were, through a mechanical system of entities, forces and processes. Although not immediately observable, such a mechanism is understood through various analogical associations to known systems. Earthquakes are explained by the causal actions of tectonic plates, extending by analogy principles of mechanics to geology. An explanatory model replicates the causal mechanism of a target system by exploiting analogical associations with a donor mechanism, based on certain observable similarities between target and donor systems (Harre 1986, Chapter 11).

\section{The Function of Scientific Prototypes}

Kuhn's theory of paradigm-driven science provoked a storm of controversy which centered on two charges. First, Kuhn failed to provide a reasonably clear conception of a paradigm, leaving the reader with a long list of possible entries (Masterman 1970). A paradigm can be a theory, law, experiment, empirical discovery, even a scientific instrument. Since the notion of paradigm is so broadly conceived, the thesis that science is paradigm-driven becomes almost vacuous 
(Shapere 1964). Second, Kuhn did not adequately show that a paradigm-shift actually improves our understanding of nature. How is our understanding of physical reality advanced, and our knowledge of nature's laws improved through commitment to paradigms? No clear answer is given in Kuhn's work (Newton- Smith 1981, Chapter V). According to one critic, Kuhn portrays science as "an irrational monster", subject to the political ideology, religious dogma and personal self-interest (Laudan 1984).

In spite of these objections, Kuhn offers the following profound insight into the character of scientific change: throughout history scientists use some discoveries as exemplars for achieving theoretical understanding beyond its intended context. Certain achievements are idealized in the service of developing other, more hypothetical, systems. In this context I recommend that talk of paradigms be abandoned in favor of prototype models. A prototype model is defined by both its descriptive and prescriptive functions. First, as a description, the prototype presumably replicates its intended scope by conceptually simulating select abstract properties of a set of phenomena. In this respect a prototype model is a kind of theoretical model, defined above. Second, as an exemplar for theories, the prototype presumably offers (prescriptively) an idealization for guiding development of other (non-prototype) models. Specifically, the conceptual content of a prototype model is extended to a poorly developed area of study. Scientists are invited to explore a new realm of conceptual possibilities, from which models can be defined, tested and tentatively accepted. The power of a prototype is not found in the logic of validation, but rather in strategies for constructing testworthy models. The utility of a prototype model cannot be explained by logical inference from past evidence to an unproven hypothesis, because no type of logical inference per se is involved in modeling. A prototype functions as a catalyst for transformation, guiding scientists in the construction of promising models.

This is revealed sometimes when scientists face an intractable problem. If a problem is immune to resolution at a particular time, scientists may employ a radical mode of transformation in the understanding of the target environment. A prototype model is used as a donor for extending its categories and relations to some problematic target. New properties are introduced and familiar ones are ignored in ways that recommend an alternative conception of the subject matter under investigation. This reformulation of the target resonates in the problemsolving strategies available to scientists: an impoverished range of possible solutions can be replaced by an alternative set of possibilities, one which contributes to a reconception of the target. A new prototype model empowers scientists with the conceptual tools for restructuring the target environment for the purpose of constructing an alternative set of possible solutions.

Prototypes are either inter-theoretic or intra-theoretic. It is precisely the analogical projections across distinct theories that render a prototype inter- 
theoretic. Transcending its immediate context of application, an inter-theoretical prototype functions as a catalyst for theoretical change in some "distant" theory. Methodologically, certain positive and negative analogies remain invariant of both the prototype and its problematic target. But neutral analogies are pivotal, since the target undergoes transformation precisely with respect to neutral relations that are defined by the donor (prototype) model. For example, the nineteenth century unification of chemistries was advanced by exploiting inter-theoretic prototypes of inorganic compounds to an understanding of organic phenomena. By 1830 inorganic chemistry was highly developed, following the discoveries of Antoine-Laurent Lavoisier, the principles of atomic combination by John Dalton, and the electrochemical theories of Sir Humphry Davy. But organic compounds were relatively unexplored at that time. Some chemists, such as J. J. Berzelius, prescribed as a methodological norm that organic compounds should be compared analogically to inorganic compounds (Brooks 1973; 1987). Which properties should be extended from chemistry to biological processes?

The French chemist August Laurent argued that both organic and inorganic compounds reduce to simple numerical ratios of atoms. The molecular structure of any substance was defined by the relative positions, arrangements, and order of constituent radicals (Kapoor 1969, 511). An accomplished crystallographer, Laurent extended principles of crystallography to the combining proportions of molecular structures. The composition of the salt lead sulfate served as an exemplar for revealing such principles. Just as the constituent elements of lead sulfate retain their original forms during chemical reaction, the organic molecule can be represented by a stable geometrical form as biological organisms develop. Laurent went on to explain both organic addition reactions and organic substitution reactions through a unitary conception of molecular structure. In particular, explanatory models for biological processes conveyed the relative positions and arrangements of constituent elements in Euclidean space. Consequently, the principles of solid geometry, which were well known in the chemistry of crystalline structures, were extended by analogy to explanations of organisms. The crystalline structure of lead sulfate functioned for Laurent as a powerful prototype for the molecular composition of organic substances, contributing to the birth of organic chemistry (Kapoor 1969).

In contrast to inter-theoretical prototypes, an intra-theoretical prototype requires that both the prototype model and its target model fall within the scope of a single theory. An intra-theoretical prototype is localized, so that the immediate impact of the prototype does not escape the confines of a particular theory. In molecular genetics for example the understanding of viruses functions prototypically for the genetic structure of cellular life. The famous Hershey- Chase experiments in 1952 focused on the replication of the bacterial virus known as phage T2. From these experiments the mode of replication for viral genes 
provided scientists with valuable insights into genome replication for many organisms. In particular, replication of the virus is far more varied than that of cells. In cellular life the hereditary molecules are typically double-stranded DNA. But viruses contain either single-stranded or double-stranded DNA, or RNA. Because of this diversity, geneticists frequently exploit the models of such replication for an understanding of analogous cellular function (Voet and Voet 1990, Chapter 32). Within molecular genetics the similarities between the bacteria phage and cellular function prompted biologists to use models of the virus as prototypes.

The distinction between intra-theoretic and inter-theoretical transformations is sensitive to scientific advances at a given time. There is no theory-neutral criterion for distinguishing the subject matters of two theories, and we cannot provide an a priori demarcation criterion between two disciplines. In all cases of prototype modeling, the promise for scientific progress is identified by extending conceptual elements from a donor system, with the effect of improving the prospects for theoretical unification.

According to most advocates of scientific analogy, every successful analogy is grounded on an interpretation of unfamiliar phenomena in terms of familiar ones. This contrast presumably establishes an epistemic asymmetry between donor and the target systems. For example, the virtues of analogy are grounded on the following factors.

(1) The causal mechanism of the donor system is known but that of the target system is unknown.

(2) The donor system enjoys a higher degree of confirmatory evidence than the confirming evidence of the target.

(3) The degree of simplicity is greater for the donor.

(4) The donor system has greater explanatory power than the target.

These four claims support, presumably, an epistemic asymmetry, according to which the donor has a higher epistemic status, as it were, than the target, prior to the analogical transformation. Based on this asymmetry, if $\mathrm{M}(\mathrm{A}, \mathrm{B})$ is the modeling relation between target $\mathrm{A}$ and donor $\mathrm{B}$, then $\mathrm{B}$ has a higher epistemic status than $\mathrm{A}$, and $\mathrm{A}$ cannot function as a donor to $\mathrm{B}$.

However, the principle of asymmetry is problematic on two counts. First, according to some advocates of analogical modeling, the asymmetry principle implies that the target system is unknown, or at least seriously flawed. But this requirement is not always realized in actual cases. Many scientific analogies occur in a context where the target model has a strong epistemic standing relative to competitors. Typically, the target model is worthy of scientific exploration, because it accounts for a wide range of phenomena, has a well-developed set of variables, or successfully predicts some behavior of the system. 
Second, the asymmetry relation is not universal, because in some cases the analogical modeling occurs "in both directions". Sometimes, scientists identify a model A as a donor model for the target B, and also identify in the same case a subset of B as a donor for a subset of A. For example, following the stunning results of explaining organic compounds in terms of electrochemical principles of inorganic chemistry, Laurent sought to reverse the direction of the analogy. While certain aspects of organic compounds were modeled on inorganic processes, other attributes of the inorganic compounds were based on models of organic phenomena (Brooke 1973).

\section{Instruments as Analogies of Nature}

One productive use of analogy can be found in the development of experimental instruments. New experimental techniques are often discovered by exploring the analogies to known phenomena. Modern instruments are designed as technological analogues to physical or chemical systems, a feature of instruments which has escaped the attention of philosophers of science. Designers try to mimic those processes of nature which can be exploited for experimental investigations of a specimen's properties.

C. T. R. Wilson designed the cloud chamber not as a particle detector, but as a meteorological reproduction of real atmospheric condensation. As Galison and Assmus document, meteorologists of the 1890's commonly designed various laboratory techniques to mimic natural occurrences (1986). Miniature versions of cyclones, glaciers and windstorms were recreated in laboratories. Towards this goal, Wilson appealed to John Aitken's dust chamber, which reproduced the effects of fogs threatening England's industrial cities at that time. Wilson incorporated the basic components of the dust chamber, such as the pump, reservoir and filter, to his cloud chamber, in order to mimic thunderstorms, coronae, and atmospheric electricity, based on analogies to atmospheric phenomena. Later, J. J. Thomson and the researchers at the Cavendish Laboratories gave the "same" instrument a new rationale. Rather than imitating cloud formations, Thomson intended to take nature apart by exploring the fundamental character of matter, in terms of electronic properties (Galison and Assmus 1986, p. 265).

How can we explain the success of modern instruments in contemporary research? A familiar answer is given by advocates of empiricist epistemologies. For example, Robert Ackermann argues that the primary function of scientific experiments in which instruments are used is to break the line of influence from theory to fact, to ground the subjectivity of instruments on the intersubjectivity of fact (1985, p. 28). Through instrumentation, the influence 
of theory is broken presumably by refining and extending human sensory capacities (1985, pp. 127-131).

However, Ackermann's rationale has little bearing on the function of modern instruments. Analytical instruments in modern chemistry, for example, are not designed to isolate the objectivity of "facts" from the subjectivity of theory, but are constructed as information processing systems. The use of modern instruments never gives experimenters a pure vision of substance in it pristine state. When using such instruments, the experimenter tries to trick the specimen into revealing its secrets through a sequence of actions and reactions at the microscopic level. The specimen is poked, dissected and disturbed, typically from the manipulating probes of radiation. In particular, an artificial energy source emits photons which impinge on a specimen. The specimen undergoes various changes of its internal dynamics, causing experimental phenomena to occur. Following such events, signals are produced, detected, enhanced and converted to a readable form. The resulting data are not always identified with perceptual experiences, contrary to the conception of data by empiricists-oriented philosophers (Bogen and Woodward 1992, 593). Typically, the experimenter reads graphic displays, digital messages, or coded charts directly from a readout device.

We should abandon the notion that scientists design modern instruments to enhance our sensory capacities to literally see the microscopic properties of substance. The chemist's closest contact to atomic processes centers on artificially- generated events, which are crafted from the union of apparatus and specimen in the process of retrieving information (Rothbart and Scherer 1997). Consider for example the chemists' use of an absorption spectrometer, designed for identifying the composition of a chemical substance. Information is produced from a series of fleeting microscopic phenomena. Radiation emitted from an energy source takes the form of discrete photons, whose function is to probe the specimen's internal dynamics. In this context a signal is understood as a quantity of energy, analogous to impulses of electromagnetic radiation from flashes of light. The analogical associations between signals and impulses of light invite scientists to extend various models of electromagnetism to absorption spectrometers. In addition to these models, models from optics, atomic theory, chemistry and geometry, function as prototypes for the design of an absorption spectrometer. In this way, an absorption spectrometer functions as an analogue to known dynamical systems, for the sake of converting experimental phenomena to information about a chemical compound (Rothbart and Slayden 1994).

A skeptic might argue that the goal of retrieving information through modern instruments is hopeless, because the apparatus may have deteriorated, the technician may be inadequately trained, or the signal may be damaged by external interference, all without the experimenter's knowledge. But in response to the skeptic, the possibility of error does not warrant the reasonable likelihood of 
error. A primary goal of various experimental techniques is to confound potential obstructions in the production of the desired signal. The skeptic-experimenter will show signs of neurosis if the instrument is repeatedly checked beyond necessity (Dretske 1981, pp. 115-116).

\section{On the Need for Metaphoric Description}

According to Kuhn, the commitment to a paradigm establishes severe restrictions on the vocabulary available to scientists at a given time. Not only are certain terms defined uniquely within the context of paradigm-driven science, but scientists are incapable of properly using terms associated with a rival paradigm. Because successful translation of vocabulary across competing paradigms is impossible, attempts by scientists to communicate with one another are always subject to a linguistic incommensurability (Kuhn 1983; Hoyningen-Huene 1993, Chapter 6).

However, Kuhn's skepticism is unwarranted. Not all communication across paradigms (read: prototypes) is threatened, but the conventional list of literal expressions associated with an old paradigm is under scrutiny. As a common response to a prototypeshift, scientists often abandon literal expressions in favor of metaphoric descriptions. The concepts of light waves, electrical How, flow of time, and information retrieval during protein synthesis are all born from metaphoric associations.

Biologists today write freely about animal-predators as economic consumers. The predator's choice of commodities reduces to an optimization problem, where the predator tries to maximize net utility. Description of natural selection is often drawn from the microeconomic models of consumer choice theory. The animal subject is rendering choices among alternative packages of commodities. In particular, three fundamental concepts of consumer choice theory, the concepts of utility, income, and price, are analogically extended to animal foraging models. Utility becomes the measure of satisfaction, interpreted as energy gained from the commodity and non-foraging time saved; income is the foraging energy and foraging time available to the subject/animal; and price is the search time for a particular prey (Stephens and Krebs 1986). Consider the following two metaphoric sentences arising from animal foraging models:

The predator foraging time and energy are searching costs charged to the predator as part of the optimization problem (Winderhalder 1983, 79).

The risk of predation to the forager and the distribution of resources are the major interacting variables that regulate consumer movement (Covich 1976, 242). 
These two metaphoric statements provide information about the predator-prey relationships, based on analogies of animals to economic consumers.

The scientists' commitment to a new prototype may strain the capacity of the conventional vocabulary to express innovative ideas. A prototype-shift may precipitate a crisis in literal vocabulary, because such vocabulary may fail to facilitate communication of new discoveries. Metaphor may be the only reasonable response to a linguistic crisis, contributing to its resolution and not its agitation.

The defining mark of a metaphor is not the falsity of some literal claim, because the negation of a metaphoric statement is not always a literal claim. Rather, metaphoric description arises from a momentary suspension of rules for literal vocabulary. What was impossible, inconceivable, and incoherent based on familiar vocabulary becomes possible, conceivable, and coherent through metaphoric redescription. Combinations of terms which were incoherent, in relation to the conventional rules of meaning, become meaningful. The semantics of a metaphor conveys an alternative realm of conceptual possibilities, through a new range of possible predicates.

At a deeper level of semantic analysis, the fundamental unit of a metaphor is a semantic field. Metaphoric meaning is a special case of conceptual combination, creating new meanings by combining concepts from ostensibly distinct semantic fields. In particular, scientists use metaphor to suggest a change of meanings of familiar terms. Possible attributes from one semantic field, called the donor field, are extended hypothetically to another field, called the target field. Following the theory of F. de Saussure, the meaning of any single expression is inseparable from its relative "placement" in a system of meanings. For example, the meaning of "blue" depends upon its position in relation to other color expressions within a field. No single color system has a priori supremacy over others, since the field of colors is dependent on the variations of language usage (Saussure 1966).

Some semantic fields are compatible with one another, permitting predication of terms from both fields. The field of domesticated animals is compatible with the field of four-legged animals, because the concept of animal may "participate" in both fields. But in other cases one field may be incompatible with another, prohibiting predication from both fields (Kittay 1987). The field of insects and the field of computers are incompatible with one another, because nothing can be members of both fields. Metaphoric meaning produces a semantic tension that arises from the juxtaposition of incompatible fields, with the effect of challenging the dictionary for literal expressions at a particular time. Every metaphoric meaning presupposes the second-order interaction of incongruous semantic fields. For example, to say that foraging time is a searching cost charged to the predator as an optimization problem implies the interaction of a field from evolutionary biology and one from macroeconomics. The animal is metaphorically 
an economic consumer who renders choices between alterative packages of commodities (Rothbart 1997, Chapter 2).

Of course, not all scientific language is metaphoric. But we cannot completely dismiss every instance of metaphor from the scientific vocabulary. Such a dismissal would imply an unwarranted bias in favor of the conventional modes of thought, associated with a particular set of scientific models. If metaphors were universally prohibited, then the language inspired by many new prototypes would be deficient. When unexpected empirical findings raise serious doubts about a familiar scientific theory, a satisfactory resolution may call for the use of metaphoric language. In some cases metaphor offers the only avenue for expressing promising but unexplored modes of thought for a particular target system. Again, the growth of scientific knowledge from use of prototypes often produces a linguistic crisis which requires the use of metaphor.

In two respects, a study of scientific analogies contributes to a compelling formulation of Kuhnian themes. First, the problematic notion of paradigm is replaced by that of a prototype model, understood through analogical associations. Second, a shift in the commitment to a scientific prototype becomes a reasonable response to severe impasses to scientific progress. Prototype modeling functions as a productive technique for scientific problem-solving through the analogical juxtaposition of distinct systems. In general, a shift in the prototype model can propel scientists to a new range of theoretical possibilities, so that the realm of possible models at the scientists' disposal can be transformed. Yet the discovery of fresh analogies does not require a monolithic overhaul of the entire scientific enterprise, as suggested in a Kuhnian' paradigm-shift. The analogical character of a scientific prototype, and the accompanying metaphoric descriptions, are often decisive in the evolution of science.

\section{References}

Ackermann, R. (1985), Data, Instruments and Theory. Princeton: Princeton University Press.

Black, M. (1962), Models and Metaphors. Ithaca: Cornell University Press.

Bogen, J. And Woodward, J. (1992), "Observations, Theories and the Evolution of the Human Spirit", Philosophy of Science 59: 590-611.

Bonner, J. (1953), „Analogies in Biology”, Svnthese 15: 275-279.

Brooke, J. (1971), „Organic Synthesis and the Unification of Chemistry-A Reappraisal”, The British Journal for the History of Science 5: 363-392.

- (1973), „Chlorine Substitution and the Future of Organic Chemistry”, Studies in the History and Philosophy of Science 4: 47-94.

- (1980), „The Chemistry of the Organic and the Inorganic”, Kagakushi (Journal of the Japanese Society for the History of Chemistry) 7: 37-60. 
- (1987), „Methods and Methodology in the Development of Organic Chemistry”, $\underline{\text { Ambix }}$

34: $147-155$.

Bunge, M. (1973), “Analogy, Simulation, and Representation”, Method, Mode, and Matter. Dordrecht-Holland: D. Reidel, pp. 114-130.

Canguilhem, G. (1963), „The Role of Analogies and Models in Biological Discovery”, in A. Crombie (ed.), Scientific Change. New York: Basic Books, pp. 507-520.

Covich A. (1976), ? Analyzing Shapes of Foraging Areas: Some Ecological and Economic Theories?, Annual review of Ecological systems 7: 235-257.

Dretske, F. (1981), Knowledge and the Flow of Information. Cambridge, Massachusetts:

The MIT Press.

Farber, E. (1950), „Chemical Discoveries by Means of Analogies”, Isis 41: 20-26. Galison, P. and Assmus, A. (1989), “Artificial Clouds and Real Particles", in Gooding,

Pinch and Schaffer (eds.), The Uses of Experiment. Cambridge: Cambridge University

Press. Hall, T. (1968), „On Biological Analogs of Newtonian Paradigms”, Philosophy of Science 35: 6-27.

Harre, R. (1970), Principles of Scientific Thinking. Chicago: University of Chicago Press.

- (1986), Varieties of Realism. Oxford: Basil Blackwell.

Hesse, M. (1966), Models and Analogies in Science. Notre Dame, Indiana: University of

Notre Dame.

- (1974), The Structure of Scientific Inference. London: Macmillan.

Hoyningen-Huene, P. (1993), Reconstructing Scientific Revolutions: Thomas S. Kuhn's

Philosophy of Science, trans. By A. T. Levine. Chicago: University of Chicago Press.

Kapoor, S. (1969), AThe Origins of Laurents Organic Classification ${ }^{\circledR}$, Isis 60: 477-527.

Kargon, R. (1969), „Model and Analogy in Victorian Science-Maxwell's Critique of the French Physicists", Journal for the History of Ideas 30: 423-426.

Kittay, E. (1987), Metaphor: Its Cognitive Force and Linguistic Structure. Oxford: Clarendon Press.

Kuhn, T. (1962), The Structure of Scientific Revolutions. Chicago, Ill.: University of Chicago Press.

- (1970), "Reflections on My Critics", in Lakatos and Musgrave (eds.)(1970), pp. 231 $-278$.

- (1977), "Objectivity, Value Judgment, and Theory Choice”, The Essential Tension: Selected Studies in Scientific Tradition and Change. Chicago: University of Chicago Press, pp. 320-339.

- (1983), "Commensurability, Comparability, Communicability", in P. Asquith and T. Nickles (eds.), PSA 1982. East Lansing, Michigan: Philosophy of Science Association, pp. 669-688.

Lakatos, I. and Musgrave, A. (eds.), (1970), Criticism and the Growth of Knowledge. Cambridge: Cambridge University Press.

Laudan, L. (1984), „Dissecting the Holist Picture of Scientific Change”, Science and Values. Berkeley: University of California Press, pp. 67-102.

Masterman, M. (1970), „The Nature of a Paradigm,” in Lakatos and Musgrave (eds.), (1970), pp. 59-90.

McGuire, J. (1970), „Atoms and the ‘Analogy of Nature': Newton's Third Rule of 
McMullin, E. (1967), „What do Physical Models Tell Us?,” in B. Van Rootselaar and J. Staal (eds.), Logic, Methodology and Philosophy of Science III: Proceedings of theThird International Congress for Logic, Methodology and Philosophy of Science.

Amsterdam: North-Holland Publishing Company, pp. 384-396.

Miller, A. I. (1986), Imagery in Scientific Thought: Creating 20th-Century Physics. Cambridge, Massachusetts: The MIT Press.

Newton-Smith, W. (1981), The Rationality of Science. New York: Routledge \& Kegan Paul.

North, J. (1980), ,Science and Analogy”, in M. Grmek, R. Cohen, and G. Cimino (eds.), On Scientific Discoveries. Vol. 34, Boston Studies in Philosophy of Science, Dordrecht: Holland, D. Reidel, pp. 115-140.

Nyhof, J. (1988), "Philosophical Objections to the Kinetic Theory", British Journal for the Philosophy of Science 39: 81-109.

Rothbart, D. (1997), Metaphors, Models and Meanings: Explaining the Growth of Scientific Knowledge. The Edwin Mellen Press.

- and Scherer, I. "Kant's Critique of Judgment and the Scientific Investigation of Matter", Hvle: An International Journal for the Philosophy of Chemistry 3: 65-80.

Rothbart, D. and Slayden, S. (1994), “The Epistemology of a Spectrometer", Philosophy of Science 61: 25-38.

Saussure, F. de (1966), Course in General Linguistics. C. Bally, A. Sechehaye, and A. Riedlinger (eds.), trans. W. Baskin. New York: Philosophical Library.

Spector, M. (1965), „Models and Theories”, British Journal for the Philosophy of Science 16: $121-142$.

Stevens, D. and Krebs, R., (1986), Foraging Theory. Princeton, New Jersey: Princeton University Press.

Swanson, J. (1966), „On Models”, British Journal for the Philosophy of Science 17: 297-311.

Voet and Voet (1990), Biochemistry. New York: John Wiley \& Sons.

Wilson (1989), "Hertz, Boltzmann and Wittgenstein Reconsidered", Studies in History and Philosophy of Science 20: 245-263).

Winterhalder, B. (1983), „Opportunity-Cost Foraging Models for Stationary and Mobile Predators", The American Naturalist 122: 73-84. 